\title{
SOME APPLICATIONS OF DIFFERENTIAL ALGEBRA TO RING THEORY
}

\author{
JOHN COZZENS AND JOSEPH JOHNSON
}

ABSTRACT. It is shown that simple noetherian $V$-domains with a unique simple module can have any global homological dimension.

In this paper some results from differential algebra are applied to produce examples of rings of linear differential operators that have some interesting ring theoretic properties. Our Theorem 1 implies that simple noetherian $V$-domains ${ }^{1}$ with a unique simple module can have any global homological dimension. Theorem 2 sharpens and extends a result found in [1].

We fix once and for all our notation:

$F=$ a differential field,

$\Delta=\left\{\delta_{1}, \cdots, \delta_{m}\right\}=$ the fundamental set of derivation operators of $F$,

$\mathfrak{D}=$ the ring of linear differential operators with coefficients in $F$,

$F\{y\}=$ the differential polynomial ring over $F$ in a single differential indeterminate $y$.

The reader may consult [3] for the definitions of all but the last of these symbols which is defined in [4].

THEOREM 1. (a) If $F$ is a universal differential field, then $\mathfrak{D}$ is a simple $V$-domain and $F$ is its unique simple module.

(b) The global homological dimension of $\mathfrak{D}$ is $m$ (whether or not $F$ is universal).

We should take note of the fact that $F$ is naturally a left $\mathfrak{D}$-module, the operation of $\mathfrak{D}$ on $F$ being given by

$$
\left(\sum a_{\theta} \theta\right)(b)=\sum a_{\theta} \theta(b)
$$

where $\theta$ runs over the set of all commutative monomials in the elements of $\Delta$.

Received by the editors October 25, 1970.

AMS 1969 subject classifications. Primary 1280, 1690.

Key words and phrases. Differential algebra, rings of linear differential operators, $V$-ring, universal differential field, global homological dimension.

${ }^{1}$ As defined in [1], a $V$-ring $R$ is a ring such that all simple left $R$-modules are injective.

(C) American Mathematical Society 1972 
To show that $F$ is the only simple $\mathfrak{D}$-module, it will suffice to show that if $I$ is a left ideal of $\mathfrak{D}$ such that $I \neq \mathfrak{D}, \operatorname{Hom}_{\mathfrak{D}}(\mathfrak{D} / I, F) \neq 0$. We need therefore to find a nonzero solution in $F$ of the system of homogeneous linear differential equations $D y=0, D \in I$.

Let $G$ be the differential subfield of $F$ generated by the coefficients of a finite generating set $S$ of $I$, and $Q$ the differential ideal of $G\{y\}$ generated by the set of linear differential polynomials $D y$ with $D$ in $S$. Then $Q$ is prime and separable over $G$. By universality, $F$ contains a generic zero $z$ of $Q$. Clearly $z$ is the required solution.

To show that $F$ is an injective $\mathfrak{D}$-module it is enough to show that any $\mathfrak{D}$-module morphism $u: I \rightarrow F$ where $I$ is a left ideal of $\mathfrak{D}$ extends to all of $\mathfrak{D}$. Thus we need to show that there exists $g$ in $F$ such that $D g=u(D)$ for all $D$ in $I$.

In general if $A$ is any polynomial ring over a field $k$ and $P$ an ideal of $A$ generated by elements $L_{t}-a_{t}$ where

(1) each $L_{t}$ is an element of $A$ homogeneous of degree 1,

(2) each $a_{t}$ is in $k$,

(3) $\sum b_{t} L_{t}=0$ with the $b_{t}$ in $k$ and almost all zero implies $\sum b_{t} a_{t}=0$, then $P$ is a prime ideal of $A$. This fact allows us to conclude that the ideal of $F\{y\}$ generated by the $D y-u(D)$ with $D \in I$ is a prime differential ideal $P$ that is separable over $F$. Because $F$ is universal, $P$ possesses a zero $g$ in $F$. Clearly $g$ satisfies the required equations.

To complete the proof of (a) we need to show that $\mathfrak{D}$ is a simple ring. Let $D$ be a nonzero element of lowest order of the two sided ideal $I$ of $\mathfrak{D}$. If $D$ were of order $r>0$, then we could fix an element $u$ of $F$ with $D(u) \neq 0$ and an easy computation shows that $D u-u D$ would be a nonzero element of $I$ of order $<r$. Hence the order of $D$ is 0 , that is $D \in F$, so that $I=\mathfrak{D}$.

To prove (b) let $M$ and $N$ be left $\mathfrak{D}$-modules. It will be shown that

(1) $\operatorname{Ext}_{\mathfrak{D}}^{n}(M, N)=0$ if $n>m$,

(2) $\operatorname{Ext}_{\mathfrak{D}}^{m}(F, \mathfrak{D}) \cong F$.

By Theorem 1 of $[2, \S 1]$ there is a spectral sequence converging to $\operatorname{Ext}_{\mathfrak{D}}^{p+q}(M, N)$ whose initial term is

$$
E_{1}^{p q}=\Lambda^{p} T^{*} \otimes_{F} \operatorname{Ext}_{F}^{q}(M, N)
$$

where in the present context $T^{*}$ is a vector space over $F$ of dimension $m$. If $p+q>m$, clearly $E_{1}^{p q}=0$ which proves (1).

To prove (2), note that since $E_{1}^{p q}=0$ if $q \neq 0$,

$$
\operatorname{Ext}_{\mathfrak{D}}^{m}(F, \mathfrak{D}) \cong H^{m}\left(\Lambda^{*} T^{*} \otimes_{F} \mathfrak{D}\right) .
$$

The formula for $d_{m}$ in Lemma 5 of $[2, \S 1]$ gives a formula for the map $\Lambda^{m-1} T^{*} \otimes_{F} \mathfrak{D} \rightarrow \Lambda^{m} T^{*} \otimes_{F} \mathfrak{D}$. From that formula we can immediately 
derive

$$
H^{m}\left(\Lambda^{\cdot} T^{*} \otimes_{F} \mathfrak{D}\right) \cong \mathfrak{D} /\left(\delta_{1} \mathfrak{D}+\cdots+\delta_{m} \mathfrak{D}\right) .
$$

This is easily seen to be canonically isomorphic to $F$ (as an abelian group).

THEOREM 2. The left $\mathfrak{D}$-module $F$ is injective if and only if every left $\mathfrak{D}$ module which is finite dimensional as a vector space over $F$ is injective.

The proof is a simple exercise if one uses the canonical isomorphism

$$
\operatorname{Hom}_{\mathfrak{D}}\left(P, \operatorname{Hom}_{F}(M, N)\right) \cong \operatorname{Hom}_{\mathfrak{D}}\left(P \otimes_{F} M, N\right)
$$

of Corollary 1 to Lemma 5 of Chapter I, §1 of [2].

Corollary. Let $F$ be an ordinary differential field. Then $\mathfrak{D}$ is a $V$ domain if and only if $F$ is an injective $\mathfrak{D}$-module.

\section{BIBLIOGRAPHY}

1. John H. Cozzens, Homological properties of the ring of differential polynomials Bull. Amer. Math. Soc. 76 (1970), 75-79. MR 41 \#3531.

2. Joseph Johnson, Extensions of differential modules over formal power series rings, Amer. J. Math. 93 (1971), 731-741.

3. - Differential dimension polynomials and a fundamental theorem on differential modules, Amer. J. Math. 91 (1969), 239-248. MR 39 \#186.

4. Ellis R. Kolchin, Galois theory of differential fields, Amer. J. Math. 75 (1953), 753-824. MR 15, 394; 1140.

Department of Mathematics, Columbia University, New York, New York 10027

Department of Mathematics, Rutgers University, New Brunswick, New JERSEY 08903 